\title{
Vacuum initial data, singularities, and cosmic censorship
}

\author{
Andrew M. Abrahams, ${ }^{*}$ Karen R. Heiderich, Stuart L. Shapiro, ${ }^{\dagger}$ and Saul A. Teukolsky ${ }^{\ddagger}$ \\ Center for Radiophysics and Space Research, Cornell University, Ithaca, New York 14853
}

(Received 1 April 1992)

\begin{abstract}
The formation of a naked singularity in a vacuum, asymptotically flat spacetime would be a clear violation of cosmic censorship. We find initial value solutions to Einstein's field equations that may lead to this behavior. We construct two families of asymptotically flat, axisymmetric vacuum solutions at a moment of time symmetry. The limiting members of these families are singular. Our first family represents a linear string of Schwarzschild black holes. We study the divergence of the gravitational tidal field outside the holes as their number along the string is increased. Our second family consists of prolate Brill gravitational wave packets. We examine the tidal field strength as the characteristic width of the wave is reduced towards zero. In both cases we find that configurations can be constructed with arbitrarily large fields that are not clothed by apparent horizons. These configurations are characterized by long, prolate concentrations of mass energy. We analyze our results in the context of the hoop conjecture.
\end{abstract}

PACS number(s): 04.20.Cv, 04.30. $+\mathrm{x}$, 97.60.Lf

\section{INTRODUCTION}

Recently, several examples of asymptotically flat spacetimes that may violate cosmic censorship have been found using numerical relativity simulations. Specifically, Shapiro and Teukolsky [1] have considered the collapse of nonrotating, axisymmetric spheroids of collisionless particles to prolate spindles. They find cases in which the tidal gravitational field diverges without the appearance of an apparent horizon. In a follow-up study [2], they found similar candidates in counter-rotating prolate configurations with sufficiently small rotation. An intriguing feature of these solutions, distinguishing them from naked singularities in spherical symmetry [3], is that the gravitational tidal field appears to diverge most quickly in the vacuum region just outside the matter.

One important question raised by these simulations is whether cosmic censorship is sensitive to the properties of matter, beyond the usual energy conditions. Although the singularities found in prolate collapse may be due to the use of a collisionless matter source, it is possible that nonlinear vacuum gravity plays an important role. Recently, Apostolatos and Thorne [4] have shown that even an infinitesimal amount of counter-rotation halts the collapse of infinite dust cylinders. These authors suggest that the key difference between their case and that of axisymmetric spheroidal collapse may be that nonlinear effects outside the matter, triggered by strong fields at the pointed ends of the spindles, might lead to the formation

\footnotetext{
${ }^{*}$ Also at Center for Theory and Simulation in Science and Engineering, Cornell University.

${ }^{\dagger}$ Also at Departments of Astronomy and Physics, Cornell University.

$\ddagger$ Also at Departments of Physics and Astronomy, Cornell University.
}

of singularities. A possibly simpler explanation is that the effective potential for the matter motion in the finite case may be sufficiently weaker than in the infinite case that it cannot halt collapse in certain situations. In any event, a very compelling case that cosmic censorship could indeed be violated would be the formation of a naked singularity in an asymptotically flat, vacuum spacetime. The appearance of a singularity in the vacuum exterior to a prolate matter spindle suggests that such spacetimes might exist. Most significantly, a vacuum spacetime with a naked singularity could not be ruled out by dismissing the physical reality of a particular type of matter as a source in general relativity.

How does one decide what types of vacuum solutions will be likely candidates for cosmic censorship violation? Interestingly, the existence or nonexistence of apparent horizons in the evolutions of collisionless matter spheriods agrees well with the criteria set forth by Thorne [5] in his "hoop conjecture." The hoop conjecture states that a horizon will exist if and only if a mass $M$ is contained in a compact region with circumference $C$ satisfying in every direction $C \lesssim 4 \pi M$. The evolution results of Ref. [1] were foreshadowed by a study [6] of sequences of momentarily static collisionless matter spheriods. This analytic work yielded results consistent with the hoop conjecture, motivating (and providing time-symmetric initial data for) the evolution calculations. In much the same spirit, this paper presents two different families of momentarily static vacuum configurations which exhibit qualitative behavior also seemingly consistent with the hoop conjecture.

Our first example involves a linear configuration of equally (coordinate) spaced black holes spanning a fixed coordinate length. We increase the number of black holes in the row to very large values while keeping the total mass constant and study the behavior of the gravitational tidal field. We also explore the existence of a common apparent horizon, encompassing all of the holes, as the length of the row is varied. Although unrealistic, this 
example represents an intriguing vacuum analogue to the collapse of collisionless matter to a prolate spindle [7]. We find that the gravitational tidal field (measured by a Riemann invariant) diverges just outside the first and last holes as the number of holes is increased. This occurs without the formation of a common apparent horizon if the string of black holes is long enough. The cutoff length for the existence of a common apparent horizon is consistent with the hoop conjecture.

Our second example involves spacetimes consisting of axisymmetric gravitational wave packets parametrized by an amplitude, a characteristic width, and a characteristic length. Sequences of time-symmetric, asymptotically flat data are constructed with a fixed characteristic length where the width is decreased while the amplitude is adjusted in order to keep the total mass constant. We examine the divergence properties of the gravitational tidal field as the width of the packet is reduced to zero. We also search for apparent horizons while varying the characteristic length of the packet. These sequences of time-symmetric slices represent a first approximation to the real, time-dependent collapse of extremely prolate gravitational wave packets. Some of our solutions should provide interesting initial data for future numerical evolutions. Accurate calculations of this sort will probably require an axisymmetric numerical relativity code with cylindrical coordinate topology (such as that used in our initial-data sequences). Briefly stated, our calculations indicate that for any arbitrarily large value of the tidal gravitational field one can always find a sufficiently long wave packet that the tidal field attains the desired strength in a region unclothed by an apparent horizon. If analogous vacuum singularities can be formed dynamically in a full evolution simulation, serious questions will be raised about the validity of cosmic censorship.

It is important to mention the possibility that event horizons may clothe the singularities in all the examples mentioned to this point even if apparent horizons do not appear on the spatial slices considered. Even in the dynamical calculations [1,2], the singularities may in principle be clothed by event horizons. However, the singularities arise and the simulations are forced to terminate before any apparent horizons appear on the maximal spatial slices used. Wald and Iyer [8] recently have demonstrated that Schwarzschild spacetime can be foliated with slices that come arbitrarily close to the singularity without trapped surfaces existing in any of the slices. Their analysis serves as a reminder that the absence of apparent horizons does not imply the absence of event horizons (though the converse is true assuming cosmic censorship). Since the examples in this paper are on initial time slices, we are restricted to searching for apparent horizons only. Ultimately, a definitive case for any violation of cosmic censorship will require timedependent simulations with a variety of time-slicing conditions.

The plan of this paper is as follows: In Sec. II we present details of our many black-hole configurations. First, in Sec. II A we outline the basic equations. Next, in Sec. II B we summarize our numerical results. Similarly, in Sec. III A we review the basic equations for our
Brill wave packets, while in Sec. III B we describe our numerical method, and in Sec. III C we present our numerical solutions and analysis.

\section{MANY-BLACK-HOLE INITIAL DATA}

In this section we consider linear strings of Schwarzschild black holes on a time-symmetric spacelike hypersurface. The black holes are spaced equally (in terms of coordinate distance) within a fixed coordinate length $L$. For fixed $L$ and fixed total mass $M=1$, we study the properties of a sequence of configurations of black holes in which the number $N$ of black holes is increased to $N=1000$. In this large- $N$ limit, we see the following limiting behavior: For strings with $L \leq 1.4$, where $L$ is measured in units of total mass, there exists a connected component of the apparent horizon which encloses the entire configuration of black holes (a common apparent horizon). However, if $L \geq 1.5$, we do not find such a common apparent horizon, but only disjoint marginally outer-trapped surfaces (see Ref. [9] for definitions) surrounding each individual mass point. Moreover, just outside these disjoint horizons, the gravitational tidal field diverges with $N$.

As shown in Ref. [10], for two black holes on a timesymmetric hypersurface, one can find up to four marginally outer-trapped surfaces (if the black holes are sufficiently close to each other): one enclosing each mass point and the other two enclosing both masses (see Fig. 1 of Ref. [10]). We call the disjoint marginally outertrapped surfaces which each enclose a single point mass disjoint apparent horizons (although, strictly, they are only apparent horizons in the absence of a marginally outer-trapped surface farther out). If a common apparent horizon exists, in addition to the disjoint apparent horizons, one can find other marginally outer-trapped surfaces (e.g., for $N=3, L=1$, one can find a total of 11 marginally outer-trapped surfaces). However, if a common apparent horizon does not exist, the only marginally outer-trapped surfaces are the disjoint apparent horizons.

For a given configuration, we are interested in finding the common apparent horizon (if it exists), the disjoint apparent horizons, and extrema of the gravitational tidal field. In addition, in order to test our results against the hoop conjecture, we calculate various circumferences of the configuration. For fixed $L$ and $M$, the number $N$ of black holes is increased to a large number and the limiting behavior is noted. It is in this large- $N$ limit that we find regions in which the gravitational tidal field diverges. However, these regions are not hidden by any apparent horizon when the string of black holes is sufficiently long.

\section{A. Basic equations}

The problem of locating marginally outer-trapped surfaces around multiple black holes at a moment of time symmetry is a straightforward generalization of the situation found for two black holes. The latter case has been investigated previously in Refs. [10] and [11] (and references therein). We summarize the basic method below. 
A metric representing $N$ point masses of mass $m_{n}$ at arbitrary spatial points $\mathbf{r}_{n}$ at a moment of time symmetry can be written [13] [in cylindrical coordinates $(\rho, \phi, z)$ ]

$$
d s^{2}=\Psi_{N}^{4}\left(d \rho^{2}+d z^{2}+\rho^{2} d \phi^{2}\right),
$$

where, in geometrized units $G=c=1$,

$$
\Psi_{N}=1+\sum_{n=1}^{n=N} \frac{m_{n}}{2 R_{n}},
$$

with $\mathbf{R}_{n}=\mathbf{r}-\mathbf{r}_{n}$, for reference point $\mathbf{r}$. When convenient, we will drop the subscript $N$ on $\Psi_{N}$ and write $\Psi$. For linear configurations of $N$ black holes of total mass $M=1$ spaced evenly throughout a fixed coordinate length $L$ along the $z$ axis centered about the origin, we take $m_{n}=1 / N$ and the source points $r_{n}$ to be

$$
r_{n}=z_{n}=-\frac{L}{2}+L \frac{n-1}{N-1}, \quad n=1, \ldots, N .
$$

All quantities will be expressed in units of total mass $M$.

As discussed in Refs. [10] and [12] (and references therein), the apparent horizon in a time-symmetric hypersurface is an external surface. The area of a candidate two-surface is proportional to the integral of $d s$, where $d s$ is given by

$$
d s^{2}=\rho^{2} \Psi^{8}\left(d z^{2}+d \rho^{2}\right) .
$$

Consequently, one sees that finding extremal surfaces is equivalent to finding the geodesics of the metric (2.4). Let us parametrize the two-surface by $\lambda$ so that $z=z(\lambda)$ and $\rho=\rho(\lambda)$ and introduce a variable $\alpha$ defined by

$$
\frac{d z}{d \lambda}=\rho^{-1} \Psi^{-4} \cos \alpha, \quad \frac{d \rho}{d \lambda}=\rho^{-1} \Psi^{-4} \sin \alpha .
$$

Note that $\alpha$ can be interpreted as the direction of the trajectory $(\rho, z)$. Then the geodesic equations for the metric (2.4) can be written as the following three first-order ordinary differential equations in the variables $\rho^{2}, z \rho^{2}$, and $\alpha \rho^{2}[11]$ :

$$
\begin{aligned}
& \frac{d\left(\rho^{2}\right)}{d \lambda}=2 \Psi^{-4} \sin \alpha \\
& \frac{d\left(z \rho^{2}\right)}{d \lambda}=\Psi^{-4}(\rho \cos \alpha+2 z \sin \alpha) \\
& \frac{d\left(\alpha \rho^{2}\right)}{d \lambda}=\Psi^{-4}\left[\cos \alpha\left(1+4 \rho \Psi_{, \rho} \Psi^{-1}\right)\right. \\
& \left.+2 \sin \alpha\left(\alpha-2 \rho \Psi_{, z} \Psi^{-1}\right)\right] .
\end{aligned}
$$

A path $(z(\lambda), \rho(\lambda))$ is a marginally outer-trapped surface if it satisfies (2.6), starts at $\lambda=0$ on the axis, and is such that $\rho(0)=\rho\left(\lambda_{F}\right)=0$ and $\dot{z}(0)=\dot{z}\left(\lambda_{F}\right)=0$, where $\lambda_{F}$ is the value of $\lambda$ where the path again intersects the axis.

As pointed out in Refs. [10] and [12], the system (2.6) must be solved numerically. We use a high-precision Runge-Kutta integrator. We start with initial conditions $\rho(0)=10^{-15}, \alpha(0)=\pi / 2$, and iterate $z(0)$ until a marginally outer-trapped surface is found. The location of the surface can be specified by $z_{0}$, the coordinate value where the surface intersects the $z$ axis. As a check on our routine, we reproduce Fig. 1 of Ref. [10], and for $N=1$ we find $z_{0}=0.500$. (Recall that the Schwarzschild radius in isotropic coordinates is at $0.5 \mathrm{M}$.)

One way to locate the common apparent horizon is to start with an initial guess of $z(0) \gg L / 2$ and integrate Eqs. (2.6) to $z=0$. The common apparent horizon occurs when $\left.\dot{\rho}\right|_{z=0}=0$, where $\cdot=d / d \lambda$. For a given iteration, then, the sign of $\left.\dot{\rho}\right|_{z=0}$ determines the value of $z(0)$ for the next iteration. Numerically, we zoom in until $|\dot{\rho}|<10^{-6}$ when $|z|<10^{-6}$. If $\left.\dot{\rho}\right|_{z=0}$ remains positive for all $z(0)$, there is no common apparent horizon; if it changes sign and becomes negative, a common horizon is present. In practice, one has to be reasonably careful in concluding that a common apparent horizon does not exist. We use the following numerical criteria: Starting at large $z(0) \gg L / 2$, we step in with step sizes of $\leq 10^{-2}$, carefully watching the behavior of $\left.\dot{\rho}\right|_{z=0}$. If $\left.\dot{\rho}\right|_{z=0} \leq 10^{-1}$, the step size is decreased by a factor of 10 and the region in question is stepped through again. If $\left.\dot{\rho}\right|_{z=0}$ falls below $10^{-2}$, the step size is again decreased by a factor of 10 or more. If $\left.\dot{\rho}\right|_{z=0}$ remains positive for all of the initial specifications of $z(0)$ up to $z_{\mathrm{DH}}$, the location where the disjoint apparent horizon around the $N$ th body crosses the $z$ axis, we conclude that there is no common apparent horizon for this configuration. (For certain configurations, e.g., $N=25, L=1.4,\left.\dot{\rho}\right|_{z=0}$ becomes as small as $\sim 10^{-5}$, but even with a step size of $10^{-6},\left.\dot{\rho}\right|_{z=0}$ remains positive.)

Disjoint apparent horizons are found using a slightly different algorithm: Starting with $\rho(0)=10^{-15}$ and $\alpha(0)=\pi / 2$ and an initial guess $z(0)$, integrate to $z_{n}$, where $z_{n}$ is the coordinate position of the $n$th black hole (around which we are looking for a disjoint apparent horizon). At $z_{n}$, record the value of $\rho$ and call it $\rho_{n 1}$. Continue integrating until the curve reverses direction and $z_{n}$ is reached again. Call the corresponding value of $\rho, \rho_{n 2}$. The disjoint apparent horizon occurs when $\left|\rho_{n 1}-\rho_{n 2}\right|=0$. For a given iteration, the sign of $\left(\rho_{n 1}-\rho_{n 2}\right)$ helps determine an improved value of $z(0)$ for the next iteration. Numerically, we zoom in until $\left|\rho_{n 1}-\rho_{n 2}\right|<10^{-8}$ when $\left|z-z_{n}\right|<10^{-8}$.

In addition to finding the apparent horizons for each configuration, we want to investigate the tidal gravitational field. Accordingly, we calculate the Riemann invariant $I={ }^{(4)} R_{\alpha \beta \gamma \delta}{ }^{(4)} R^{\alpha \beta \gamma \delta}$. For time symmetry (i.e., zero extrinsic curvature $K_{i j}=0$ ), $I$ (in vacuum) is given, in Cartesian coordinates, by

$$
\begin{aligned}
I= & { }^{(4)} R_{\alpha \beta \gamma \delta}{ }^{(4)} R^{\alpha \beta \gamma \delta}=8^{(3)} R_{i j}{ }^{(3)} R^{i j} \\
= & 192 \Psi^{-12} \Psi_{, i} \Psi_{, j} \Psi^{, i} \Psi^{, j}-192 \Psi^{-11} \Psi_{, i j} \Psi^{, i} \Psi^{, j} \\
& +32 \Psi^{-10} \Psi_{, i j} \Psi^{, i j},
\end{aligned}
$$

where Greek indices run from 0 to 3, Latin indices run from 1 to 3 , and summation is understood. In particular, we find the value and location of $I_{\max }$, the maximum of $I$.

To relate our results to Thorne's hoop conjecture, we compute the equatorial and polar circumferences of the common apparent horizon, as well as its surface area, 


$$
\begin{aligned}
& \frac{\mathcal{C}_{\mathrm{eq}}^{\mathrm{AH}}}{2 \pi(2 M)}=\left.\frac{1}{2} \rho \Psi^{2}\right|_{z(\lambda)=0}, \\
& \frac{\mathcal{C}_{\mathrm{pol}}^{\mathrm{AH}}}{2 \pi(2 M)}=\frac{1}{\pi} \int_{\lambda=0}^{\left.\lambda\right|_{z=0}} \Psi^{2}\left(\frac{d \rho^{2}}{d \lambda}+\frac{d z^{2}}{d \lambda}\right)^{1 / 2} d \lambda, \\
& \frac{\mathcal{A}}{4 \pi(2 M)^{2}}=\frac{1}{4} \int_{\lambda=0}^{\left.\lambda\right|_{z=0}} \rho \Psi^{4}\left(\frac{d \rho^{2}}{d \lambda}+\frac{d z^{2}}{d \lambda}\right)^{1 / 2} d \lambda=\frac{1}{4} \lambda,
\end{aligned}
$$

where the last equality follows from (2.4). As a test, for $N=1$ we find $e_{\mathrm{eq}}^{\mathrm{AH}} / 4 \pi M=1.000, e_{\mathrm{pol}}^{\mathrm{AH}} / 4 \pi M=1.000$, and $\mathcal{A} / 16 \pi M^{2}=1.000$.

To gauge the hoop conjecture, we calculate the proper lengths of geodesics of various orientations of the metric (2.1) that enclose the entire configuration. We find the geodesics that give the minimum proper lengths for both polar and latitudinal orientations. We refer to these geodesics as "minimal geodesics" and the corresponding circumferences $e^{\text {min }}$ as "minimal circumferences." The minimal latitudinal geodesic will be a circle in the $(\rho, \phi)$ plane. Note that the minimal latitudinal geodesic need not occur in the equatorial $z=0$ plane: For example, for $N$ even, the minimal geodesic in the $z=0$ plane, $\left.\mathcal{C}_{\text {lat }}^{\min }\right|_{z=0}$, is zero, which clearly does not correspond to a geodesic that "encloses the entire configuration." Thus, to locate the minimal latitudinal geodesic, we must find the minimal circumference $\left.\bigodot_{\text {lat }}^{\min }\right|_{z}=\left.\frac{1}{2} 4 \pi M \rho \Psi^{2}\right|_{z} \quad$ [cf. Eq. (2.8)] associated with $z$ coordinates between 0 and $L / 2$ and choose the largest. In practice, we find that $\mathcal{C}_{\text {lat }}^{\min }$ occurs for $N$ odd at $z=0$ and for $N$ even at $z=z_{N / 2}$; i.e., the minimal latitudinal geodesic essentially occurs in the $z=0$ plane, and so we write (for consistency of notation) $\mathcal{C}_{\mathrm{eq}}^{\min }$ instead of $\mathcal{C}_{\text {lat }}^{\min }$. As a test, for $N=1$, we find that $\mathcal{C}_{\mathrm{eq}}^{\mathrm{min}} / 4 \pi M=1.000$ at $z=0$.

The minimum longitudinal or polar circumference $\mathcal{C}_{\text {pol }}^{\text {min }}$ can be taken to be a geodesic of the metric (2.1) that lies in the $\phi=$ const plane. To determine $\mathcal{C}_{\text {pol }}^{\text {min }}$, one solves the geodesic equations of (2.1), which we write [in analogy with Eqs. (2.6)]

$$
\begin{aligned}
& \frac{d \rho}{d \lambda}=\Psi^{-2} \sin \alpha, \\
& \frac{d z}{d \lambda}=\Psi^{-2} \cos \alpha, \\
& \frac{d \alpha}{d \lambda}=2 \Psi^{-3}\left(\Psi_{, \rho} \cos \alpha-\Psi_{, z} \sin \alpha\right),
\end{aligned}
$$

with initial conditions $\rho(0)=0$, varying $z(0)$ and $\alpha(0)$ until the solution that gives the minimum value of the right-hand side of (2.9) is found. Numerically, we zoom in until the circumferences associated with consecutive iterations differ by less than $10^{-6}$. We find that (up to our numerical accuracy) $\bigodot_{\text {pol }}^{\min }$ corresponds to the initial choice of $\alpha(0)=\pi / 2$. Moreover, equivalent values of $\mathcal{C}_{\text {pol }}^{\text {min }}$ (up to numerical accuracy) are obtained using the algorithm described above to locate common apparent horizons. For $N=1$, we find $e_{\text {pol }}^{\min } / 4 \pi M=1.000$ at $\alpha=\pi / 2$.

\section{B. Discussion}

We have extensively investigated the properties of linear configurations of black holes with coordinate lengths in the range $1 \leq L \leq 2$ with the number of black holes in the range $2 \leq N \leq 1000$. In addition, configurations with lengths of up to $L=8$ and with up to $N=10^{8}$ black holes have been probed less intensively. We search for common apparent horizons, disjoint apparent horizons around the $N$ th point mass, minimal circumferences, and maxima of the gravitational tidal field. Of particular interest is the limiting behavior of these parameters as $N$ becomes large. Illustrated in Fig. 1 are representative configurations with the common and disjoint apparent horizons and the minimal circumferences drawn in. Note the small variations in the location of the
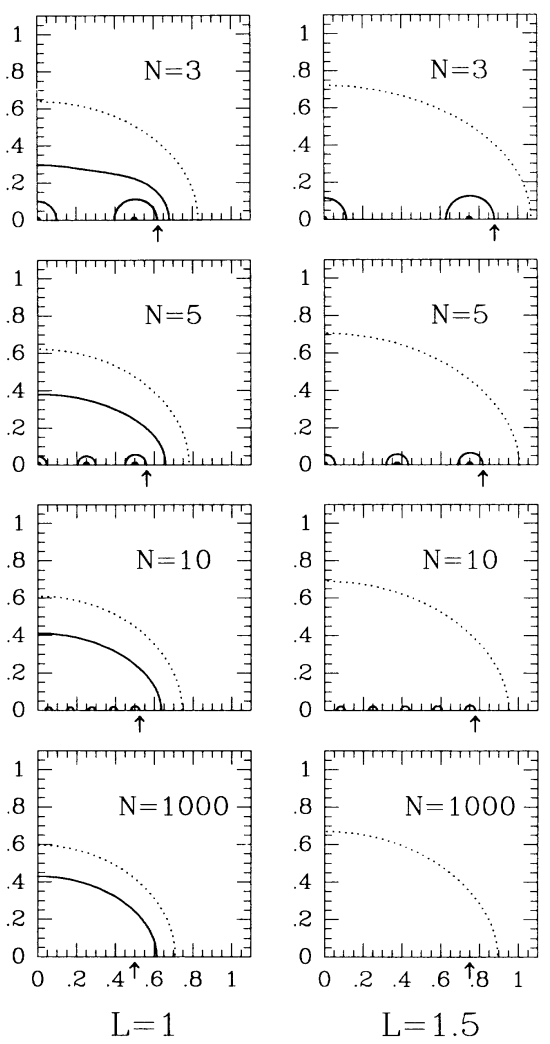

FIG. 1. Representative sample of black-hole configurations. Strings of $N=3,5,10$, and 1000 black holes are distributed evenly throughout coordinate lengths of $L=1$ (first column) and $L=1.5$ (second column). The horizontal axis is the positive $z$ direction, and the vertical axis is the $\rho$ direction. The solid lines show marginally outer-trapped surfaces, and the dotted lines show minimal polar circumferences. Surrounding individual point masses are disjoint apparent horizons (although they are too small to appear in the figure for $N=1000$ ). For configurations with $L \leq 1.4$, there is also a common apparent horizon whenever $N \gtrsim$ a few. (For $L=1$ we find common apparent horizons when $N \geq 3$.) For configurations with $L \geq 1.5$, however, we do not find common apparent horizons for any $N$ up to 1000 . The arrows indicate the location of $I_{\max }$ (which always lies outside the disjoint apparent horizon, although the resolution of the picture is not fine enough to show this). 
apparent horizons, minimal circumferences, and $I_{\max }$ as $N$ changes from 10 to 1000 . The values of some of the calculated parameters for strings of $N=1000$ black holes are tabulated in Table I.

In the limit as $N \rightarrow \infty$, the sum defining $\Psi_{N}$,

$$
\Psi_{N}=1+\sum_{n=1}^{n=N} \frac{1}{2 N\left[\rho^{2}+\left(z-z_{n}\right)^{2}\right]^{1 / 2}}
$$

[cf. Eq. (2.2)], with $z_{n}$ given by Eq. (2.3), converges. Indeed, the limit $\Psi_{\infty}=\lim _{N \rightarrow \infty} \Psi_{N}$ can be shown (using a Euler-Maclaurin formula) to be equivalent to the potential obtained by considering a (time-symmetric) uniform finite line "mass distribution" of length $L$ and constant mass density $1 / L$

$$
\begin{aligned}
\lim _{N \rightarrow \infty} \Psi_{N} & =1+\frac{1}{2 L} \int_{-L / 2}^{L / 2} d z^{\prime} \frac{1}{\left[\rho^{2}+\left(z-z^{\prime}\right)\right]^{1 / 2}} \\
& =1+\frac{1}{2 L} \ln \left[\frac{z+L / 2+\left[\rho^{2}+(z+L / 2)^{2}\right]^{1 / 2}}{z-L / 2+\left[\rho^{2}+(z-L / 2)^{2}\right]^{1 / 2}}\right] .
\end{aligned}
$$

This is, in fact, in complete analogy with the electrostatic potential for a finite line of uniform charge density (the case of a finite line of uniform mass density was investigated in Ref. [14]). We expect to see this limiting behavior in our large- $N$ sequences. The rate at which the parameters approach a limiting value is illustrated in Fig. 2 for the particular case of $\mathcal{C}_{\text {pol }}$. Similar rates of convergence are exhibited by most of the other parameters.

As noted in Refs. [10] and [12] (and references therein), for two black-hole configurations there is a critical length $L_{c_{N=2}}$ such that if $L \leq L_{c_{N=2}}$, there exists a common apparent horizon, while if $L>L_{c_{N=2}}$ there does not. In Ref. [12] it was found that $L_{c_{N=2}} \sim 0.77$ (in our units). Referring to Table I, for $N=3$, we find that $1 \leq L_{c_{N=3}}<1.1$; for $N=4$, we find $1.1 \leq L_{c_{N=4}}<1.2, \ldots ; \quad$ and for
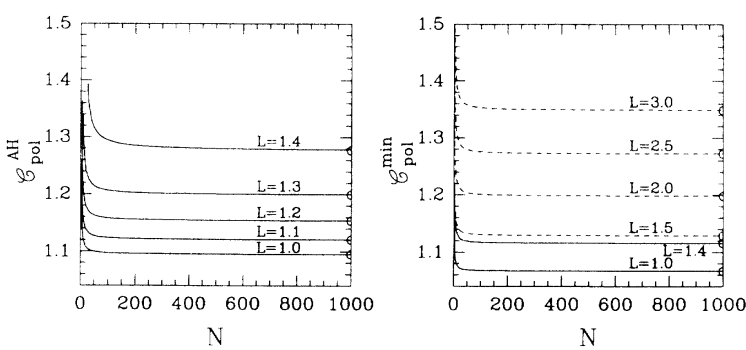

FIG. 2. Polar circumference of the common apparent horizon (left) and of the minimal circumference (right) is plotted as a function of the number of black holes $N$ for various string lengths $L$. As $N$ becomes large, $\mathcal{C}_{\text {pol }}$ approaches a limiting value. The open half circles indicate the values obtained in the $N \rightarrow \infty$ limit. The values of $\mathcal{C}_{\mathrm{pol}}^{\mathrm{AH}}$ and $\mathcal{C}_{\mathrm{pol}}^{\mathrm{min}}$ for $N=1000$ are recorded in Table $\mathbf{I}$.

$N=9,10, \ldots, 26$, we find $1.4 \leq L_{c_{N=9, \ldots, 26}}<1.5$. However, for $L \geq 1.5$, we do not find a common apparent horizon for any $N$ (up to $N=1000$ or in the limit as $N \rightarrow \infty$ ). Hence the critical length for $N>26, L_{c_{N}>26}$, lies in the range $1.4<L_{c_{N>26}}<1$. 5. Strings of black holes of coordinate length 1.5 or longer are not enclosed by a common apparent horizon.

The existence or nonexistence of a common apparent horizon is qualitatively consistent with Thorne's hoop conjecture. Referring to Table I and Fig. 2, one sees that if any minimal circumference $e^{\text {min }}$ associated with a string of black holes obeys $e^{\text {min }} / 4 \pi M \lesssim 1.116$, the configuration will be surrounded by a common apparent horizon. However, if the corresponding circumference is such that $e^{\min } / 4 \pi M \gtrsim 1.129$, the configuration will not be enclosed by a common apparent horizon: One observes a marked cutoff in the minimal circumferences of strings having a common apparent horizon as compared with strings that do not.

TABLE I. Properties of strings of $N=1000$ black holes are given. We list the coordinate length $L$ of the configuration and whether or not it is surrounded by a common apparent horizon. (We have checked up to $N=1000$ as well as the $N \rightarrow \infty$ limit.) Also given are the equatorial and polar circumferences of the common apparent horizon (if it exists) as well as its surface area, and the minimum equatorial and polar circumferences surrounding the string. In the last two columns, we list the value of the

\begin{tabular}{|c|c|c|c|c|c|c|c|c|}
\hline$L / M$ & $\begin{array}{c}\text { Apparent } \\
\text { horizon? }\end{array}$ & $\mathcal{C}_{\mathrm{eq}}^{\mathrm{AH}} / 4 \pi M$ & $\mathcal{C}_{\mathrm{pol}}^{\mathrm{AH}} / 4 \pi M$ & $\mathcal{A} / 16 \pi M^{2}$ & $\begin{array}{c}\mathcal{C}_{\mathrm{eq}}^{\min } / 4 \pi M \\
(N=1001) \\
\end{array}$ & $\mathcal{C}_{\mathrm{pol}}^{\min } / 4 \pi M$ & $I_{\max } / M^{-4}$ & $\begin{array}{c}z_{\max } / M \\
-z_{\mathrm{DH}} / M \\
\end{array}$ \\
\hline 1 & yes $(N \geq 3)$ & 0.853 & 1.095 & 0.994 & 0.00778 & 1.067 & $1.50 \times 10^{9}$ & $5 \times 10^{-7}$ \\
\hline 1.1 & yes $(N \geq 4)$ & 0.823 & 1.121 & 0.991 & 0.00716 & 1.079 & $2.02 \times 10^{9}$ & $5 \times 10^{-7}$ \\
\hline 1.2 & yes $(N \geq 5)$ & 0.789 & 1.154 & 0.987 & 0.00664 & 1.091 & $2.63 \times 10^{9}$ & $6 \times 10^{-7}$ \\
\hline 1.3 & yes $(N \geq 9)$ & 0.747 & 1.200 & 0.981 & 0.00621 & 1.103 & $3.34 \times 10^{9}$ & $6 \times 10^{-7}$ \\
\hline 1.4 & $\operatorname{yes}(N \geq 26)$ & 0.691 & 1.279 & 0.972 & 0.00584 & 1.116 & $4.16 \times 10^{9}$ & $6 \times 10^{-7}$ \\
\hline 1.5 & no & & & & 0.00552 & 1.129 & $5.07 \times 10^{9}$ & $6 \times 10^{-7}$ \\
\hline 1.6 & no & & & & 0.00523 & 1.142 & $6.08 \times 10^{9}$ & $6 \times 10^{-7}$ \\
\hline 1.7 & no & & & & 0.00499 & 1.156 & $7.12 \times 10^{9}$ & $6 \times 10^{-7}$ \\
\hline 2 & no & & & & 0.00439 & 1.198 & $1.11 \times 10^{10}$ & $6 \times 10^{-7}$ \\
\hline
\end{tabular}
maximum of the Riemann invariant $I_{\max }$ and the coordinate separation between the location of $I_{\max }$ and the location of the disjoint apparent horizon around the $N$ th black hole. 
Finally, we note the behavior of the gravitational tidal field as measured by the Riemann invariant $I$ defined in Eq. (2.7b). For a given configuration, $I$ peaks sharply on the $z$ axis at a coordinate location of $z \gtrsim L / 2$ (and $z \lesssim-L / 2$ ). We denote the value of this maximum by $I_{\max }$ and its coordinate value by $z_{\max }$. The rate at which $I_{\text {max }}$ increases as a function of increasing $N$ is seen in Fig. 3: As $N$ becomes large, $I_{\max }$ diverges. For example, for a single black hole of mass $M$, the maximum of $I$ outside the apparent horizon is $\left.I\right|_{r=0.05 M}=0.75$ (in units of $M^{-4}$ ), whereas for a string of 1000 black holes with total mass $M$ and with, say, $L=1.5, I_{\max } \sim 5 \times 10^{9}$. Additionally, for specified $N, I_{\max }$ increases in value as $L$ becomes larger. Moreover, for all configurations investigated, $I_{\max }$ occurs just outside the disjoint apparent horizon of the $N$ th black hole (cf. Table I). Although $z_{\max }$ and the coordinate location where the disjoint apparent horizon of the $N$ th black hole intersects the axis, $z_{\mathrm{DH}}$, differ by only $z_{\max }-z_{\mathrm{DH}} \sim 5 \times 10^{-7}$, this is within our numerical accuracy (which is here $\sim \pm 1 \times 10^{-7}$ ). For strings of black holes of lengths $L \geq 1.5$, this singularity is not hidden by a common apparent horizon.

It is interesting to compare these results with those obtained by Shapiro and Teukolsky [2] for the collapse of prolate spheroids of collisionless matter to form spindles. For sequences of black-hole strings, the tidal gravitational field diverges at the ends of the strings, just outside the last black hole. Similarly, in the prolate collapse, it is at the holes of the prolate spindle, in the vacuum region just outside the matter, where the gravitational tidal field appears to diverge most rapidly. In each of these cases, sufficiently elongated configurations can be found in which these regions of diverging gravitational tidal field are not enclosed by apparent horizons. In prolate spheroid collapse, it is always possible that these singu-

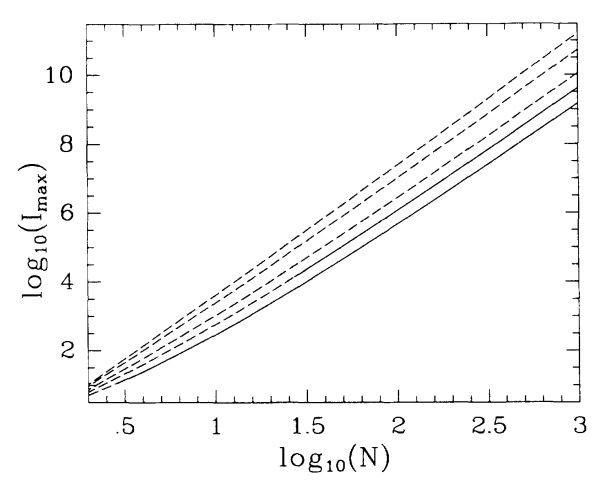

FIG. 3. Maximum of the Riemann invariant $I$ (in units of $M^{-4}$ ) on the $z$ axis at $z>L / 2$ is plotted as a function of the number of black holes in the string for various string lengths. The solid lines correspond to string lengths $L \leq 1.4$ for which there is a common apparent horizon. The dashed lines correspond to string lengths $L \geq 1.5$ for which a common apparent horizon could not be found. Data are shown for string lengths of, from bottom to top, $L=1,1.4,2,4$, and 8 . For fixed $L$, as the number of black holes in the string is increased, $I_{\max }$ increases. For fixed $N$, as $L$ increases, the corresponding value of $I_{\max }$ increases. larities are due to the choice of collisionless matter. However, since we obtain similar behavior for prolate vacuum time-symmetric sequences, it seems more likely that this behavior is due to a fundamental property of general relativity itself.

\section{BRILL WAVE INITIAL DATA}

Axisymmetric, pure-wave spacetimes are the simplest asymptotically flat generalization of the Einstein-Rosen infinite cylindrical waves [15]. These gravitational waves have been considered previously in a number of contexts. Brill [16] used the time-symmetric solution of Weber and Wheeler [17] to explore the energy of an initial-data slice in general relativity. Eppley [18] numerically solved the initial-value problem for such waves and probed for apparent horizons. Numerical evolutions of quadrupolar axisymmetric waves (both initially time symmetric and time asymmetric) have been performed [1,19], and in these cases the imploding wave either passes through itself at the origin (perhaps interacting nonlinearly) or forms a black hole. Recently, Beig and Ó Murchadha [20] have shown that certain (nonsingular) sequences of time-symmetric, vacuum, asymptotically flat initial data contain trapped surfaces when the field approaches the strong-field limit of general relativity. Here we explicitly construct vacuum initial-data sequences that do not have apparent horizons even as the field strength increases without bound.

\section{A. Basic equations}

Following Brill [16], we assume a spatial line element of the form

$$
d l^{2}=\psi^{4}\left[e^{2 \eta}\left(d \rho^{2}+d z^{2}\right)+\rho^{2} d \phi^{2}\right],
$$

appropriate for nonrotating spacetimes. The metric variables are the conformal factor $\psi$ and a function representing the transverse field $\eta$. This gauge is often referred to as quasi-isotropic because the Schwarzschild solution (in spherical coordinates) written in this gauge takes its isotropic coordinate form. At a moment of time symmetry $\left(K_{i j}=0\right)$, the vacuum Hamiltonian constraint in axisymmetry becomes [16]

$$
\Delta^{f} \psi=\frac{\partial^{2} \psi}{\partial \rho^{2}}+\frac{1}{\rho} \frac{\partial \psi}{\partial \rho}+\frac{\partial^{2} \psi}{\partial z^{2}}=-\frac{1}{4} \psi\left(\frac{\partial^{2} \eta}{\partial \rho^{2}}+\frac{\partial^{2} \eta}{\partial z^{2}}\right),
$$

where $\Delta^{f}$ is the flat-space, three-dimensional Laplacian.

We choose a simple functional form for the transverse-field function $\eta$ that allows for very nonspherical, axisymmetric, equatorial phase-symmetric wave packets:

$$
\eta=A \rho^{2} \exp \left[-\left(\frac{\rho^{2}}{\lambda_{\rho}^{2}}+\frac{z^{2}}{\lambda_{z}^{2}}\right)\right],
$$

where the constants $\lambda_{\rho}$ and $\lambda_{z}$ are characteristic wavelengths. This function satisfies the regularity conditions on the axis: 


$$
\eta=0 \text { and } \eta_{, \rho}=0 \text { at } \rho=0 .
$$

The function $\eta$ clearly falls off faster than $r^{-2}$ at large spherical radius $r$ and thus satisfies the asymptotic flatness requirement.

The inner boundary conditions on the conformal factor are regularity on the axis,

$$
\psi_{, \rho}=0 \text { at } \rho=0,
$$

and reflection symmetry on the equator,

$$
\psi_{, z}=0 \text { at } z=0 \text {. }
$$

At large radius the conformal factor takes a form dictated by asymptotic flatness,

$$
\psi=1+\frac{M}{2 r}+\cdots
$$

where $M$ is the interior mass. This requirement can be enforced as a Robin condition without reference to $M$ :

$$
\frac{\partial \psi}{\partial r}+\frac{\psi-1}{r}=0
$$

Given $\eta$, one solves the Hamiltonian constraint (3.2) for $\psi$ subject to the above boundary conditions. From this solution the total mass $M$ can be computed using Brill's [16] original explicitly positive-definite expression

$$
\begin{aligned}
M_{B} & =\frac{1}{2 \pi} \int d V(\nabla \ln \psi)^{2} \\
& =\frac{1}{2 \pi} \int d V\left[\left(\frac{\partial \ln \psi}{\partial \rho}\right)^{2}+\left[\frac{\partial \ln \psi}{\partial z}\right]^{2}\right],
\end{aligned}
$$

where $d V=2 \pi \rho d \rho d z$. Equivalently, we can compute the Arnowitt-Deser-Misner (ADM) mass [21], using Gauss' law to rewrite it as a volume integral:

$M_{\mathrm{ADM}}=-\frac{1}{2 \pi} \int d V \Delta^{f} \psi=\frac{1}{8 \pi} \int d V \psi\left(\frac{\partial^{2} \eta}{\partial \rho^{2}}+\frac{\partial^{2} \eta}{\partial z^{2}}\right)$,

where the second form is obtained using Eq. (3.2). Because of the rapid falloff of the transverse part of the field mentioned above the three mass indicators in Eqs. (3.7), (3.9), and (3.10) should all yield the mass of the slice when evaluated at large radius. As a measure of the strength of the tidal gravitational field, we compute an invariant of the Riemann tensor [see Eq. (2.7a) for the general form]. To avoid algebraic and coding errors, we used a symbolic manipulation package [22] on the pure-wave metric (3.1).

\section{B. Numerical method}

Standard second-order finite-difference schemes that automatically enforce regularity at the axis are used for the numerical solution of the (linear) Hamiltonian constraint equation (3.2). Typically, we used cylindricalcoordinate numerical grids with 140 zones in both the $\rho$ and $z$ directions. The innermost grid zone in each direction was placed at a small fraction of the corresponding characteristic wavelength. The remaining zones in each direction were geometrically spaced to the outer bound- ary, normally placed at $150 \mathrm{M}$. For improved accuracy in computing integrals, we generate the grid in terms of the variable $x=\rho^{2}$ rather than $\rho$ so that $\pi d x d z$, the coordinate volume element, locally has slow variation.

Our initial-data sequences are generated as follows. Starting with equal characteristic wavelengths $\lambda_{z}$ and $\lambda_{\rho}$, we choose a value for the amplitude $A$, solve the Hamiltonian constraint Eq. (3.2), and compute the ADM mass. We then vary the value of $A$, solve Eq. (3.2), and compute the mass until it converges to the desired value, $M=1$. Bisection is used to find the appropriate amplitude (to a fractional precision of $10^{-6}$ ) that yields $M=1$. We then compute the invariant $I$ at each grid point. The characteristic width $\lambda_{\rho}$ is then reduced by a small fraction, and the process is repeated until an entire prolate sequence of wave packets is generated. A similar method can be used for generating oblate sequences where the characteristic width $\lambda_{\rho}$ is fixed and $\lambda_{z}$ varied. Each solution of the Hamiltonian constraint involves a sparse matrix inversion, which is accomplished using the University of Waterloo's Sparspak software. We search for apparent horizons in the data sets using the method detailed in Ref. [23].

Several tests and confidence checks have been performed to verify the accuracy of this code. A convergence test (using grid resolutions between $35 \times 35$ and $280 \times 280$ ) confirmed that our finite-difference scheme was second-order accurate. Comparison of our different mass indicators was another good check. The ADM mass as computed by the integral Eq. (3.10) agreed with the mass read off from the field [see Eq. (3.7)] at large radii to a small fraction of a percent in all cases. The difference was always less than the actual inaccuracy of our ADM mass caused by our evaluating it at finite outer radius (this error drops off as $r^{-1}$ ). Equation (3.10) is considerably more accurate numerically than the Brill mass [Eq. (3.9)] for our calculation as it involves only the conformal factor and the analytically known twodimensional flat-space Laplacian of the transverse-field function $\eta$. In contrast, Eq. (3.9) requires numerical second derivatives of the conformal factor. For this reason, although the two mass integrals give nearly identical results when $\lambda_{\rho}$ and $\lambda_{z}$ are $\gtrsim 10^{-1}$ (fractional differences around $0.1 \%$ ), when one or both of these wavelengths is very small, the grid is severely stretched so that the derivatives of $\psi$ become less accurate near the outer $\rho$ boundary of the grid. For the most extreme cases, where $\lambda_{\rho} \sim 10^{-5}$, the mass difference can be as much as $2.5 \%$, though the solution accuracy in the region of interest is considerably higher. We also checked that in the linear regime the mass scales with the wave amplitude as $M \sim A^{2}$ and find that the relation holds at the $1 . \%$ accuracy level up to amplitudes of $A \simeq 10^{-3}$.

\section{Numerical results}

In this section we present some of the results of our numerical study of pure-wave initial-data sets. We give a few representative solutions and a parameter-space survey that illustrates some general trends. First, we demonstrate that our initial-data sequences do indeed show a singular behavior. In Fig. 4 we show three prolate se- 


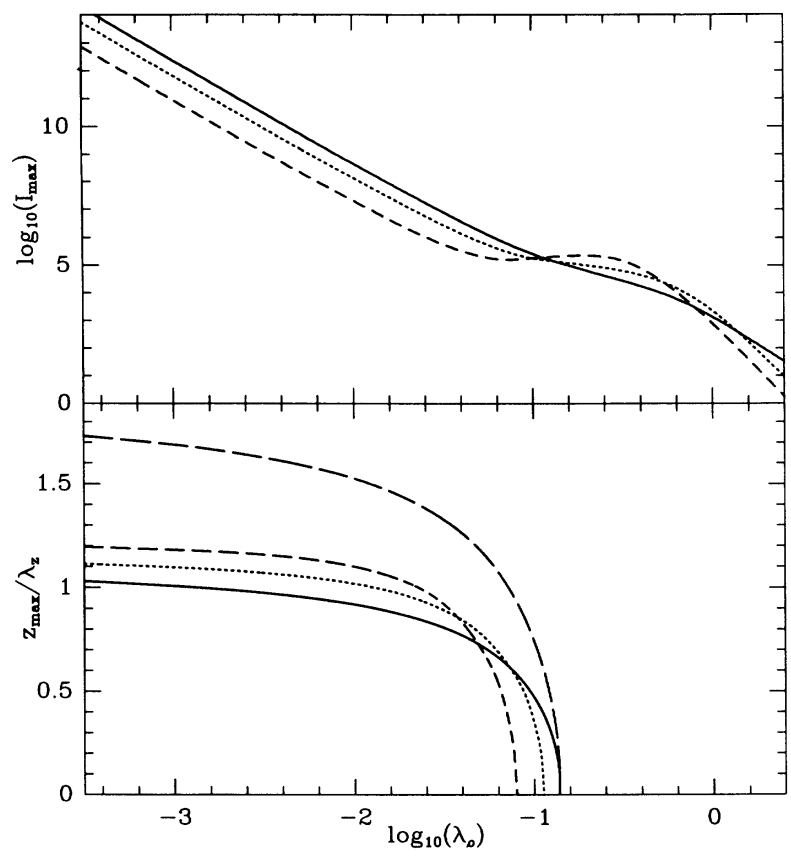

FIG. 4. Maximum value of the Riemann invariant $I$ in units of $M^{-4}$ is probed in three prolate sequences of Brill waves. In the top frame, the maximum value is shown as a function of the characteristic width of the wave. In the bottom frame, the $z$ axis position of the maximum is plotted as a function of the characteristic width. Data are shown for three values of characteristic length $\lambda_{z}=2.0$ (solid curve), $\lambda_{z}=1.0$ (dotted curve), and $\lambda_{z}=0.5$ (short-dashed curve). In the bottom frame, the long-dashed curve shows the proper distance along the axis from the origin to $z_{\max }$ for the $\lambda_{z}=2.0$ sequence.

quences in which the characteristic coordinate length of the waves $\lambda_{z}$ is held fixed, while the characteristic coordinate width $\lambda_{\rho}$ is varied by a factor of $\geq 10^{3}$. For each solution we search for the maximum value of the Riemann invariant and plot it as a function of $\lambda_{\rho}$. After a transition region, at approximately $\lambda_{\rho} \simeq 0.1 \lambda_{z}$, each curve diverges, closely following the power law $I_{\max } \propto \lambda_{\rho}^{-4}$. Even the $\lambda_{z}=0.5$ sequence, which has a region of decreasing $I_{\max }$ with decreasing $\lambda_{\rho}$, quickly approaches this relation. As one might expect, the most prolate sequence, $\lambda_{z}=2.0$, shows the greatest $I_{\max }$ in the powerlaw regime. These results clearly indicate that the field can be made arbitrarily strong if the characteristic width can be made arbitrarily small. We have constructed configurations with $I_{\max }$ as large as $10^{20}$.

Also, in Fig. 4, we examine how the positions of the maxima vary with the characteristic width. In a typical prolate sequence when $\lambda_{z}$ and $\lambda_{\rho}$ are comparable, the maximum value of $I$ is found very close to the origin. When $\lambda_{\rho}$ is decreased, the coordinate position of the maximum moves up the axis (because of equatorial plane symmetry, there is another maximum the same distance below the equator), and as $\lambda_{\rho}$ becomes very small, this coordinate location asymptotes to a finite value, usually $z_{\max } \simeq \lambda_{z}$. The movement away from $z_{\max } \simeq 0$ seems to coincide fairly closely with the transition of $I_{\max }$ into the power-law regime. To test if this is just a coordinate effect, we also show the proper half-length of the configuration, computed by integrating Eq. (3.1) from the origin up to $z_{\max }$ along the axis. Although the numerical values of coordinate and proper distance diverge (not surprisingly) as the field gets strong, the qualitative behavior is similar. It is possible that the proper length of the configurations (measured from maximum to maximum) grows slowly, rather than asymptoting to a finite value, as $\lambda_{\rho} \rightarrow 0$, but it certainly does not decrease.

Note that we are largely restricting our attention to the prolate sequences as these are the ones that best emulate a true evolution, i.e., implosion onto the axis. Sufficiently prolate wave packets might be expected to evolve similarly to infinite cylindrical waves $[15,24]$ in certain regimes. Additionally, the divergence characteristics of the prolate sequences seem to make them a more straightforward candidate for cosmic-censorship violation than their oblate counterparts. In contrast with the results shown in Fig. 4, in oblate sequences (fixing $\lambda_{\rho}$ and reducing $\lambda_{z}$ toward zero), the coordinate position of $I_{\max }$ moves out from the origin initially along the axis, and then returns to the origin and moves out along the equator, but reverses as the field strength increases, asymptoting to a finite proper distance from the axis. If both characteristic wavelengths are sufficiently small, the maxima will move away from both the equator and the axis. Also, $I_{\max }$ diverges as $I_{\max } \propto \lambda_{z}^{3}$ instead of the fourth power seen in the prolate sequences. As in the case of collisionless matter, prolate configurations of mass energy are far more effective in generating singular fields than oblate configurations.

The main difference between the prolate and oblate sequences can be clarified by examining the behavior of the wave amplitude $\boldsymbol{A}$ [see (3.3)]. For the prolate sequences, the amplitude grows rapidly: $A \propto \lambda_{\rho}^{-2}$ as $\lambda_{\rho} \rightarrow 0$; in the oblate sequences, the amplitude falls off as $\lambda_{z}^{\mathrm{P} / 2}$ as $\lambda_{z} \rightarrow 0$. This can be understood by looking at the ADM mass density [Eq. (3.10)]. Basically, in the prolate sequences, the amplitude has to increase quadratically in $1 / \lambda_{\rho}$ to counteract the quadratic decrease in the volume over which $\eta$ is appreciable ( $\psi$ is roughly independent of $\lambda_{\rho}$ in this limit). In the oblate sequences, the two-dimensional Laplacian of $\eta$ goes as $A / \lambda_{z}^{2}$ in the limit $\lambda_{z} \rightarrow 0$, and so the amplitude must decrease to compensate for the changing volume element. In this limit, in the region that contributes significantly to the mass integral, the conformal factor approaches a profile of the form $\psi \sim c\left(1-z^{1 / 2}\right)$, where $c$ is a constant independent of $\lambda_{z}$. This explains the $\frac{1}{2}$ power law found for $A$ in these sequences. Note that the scalings found numerically for $A$ are consistent with the divergence of the Riemann invariant. For the prolate sequences, the biggest terms in $I$ on the axis should be proportional to $(\eta, \rho \rho)^{2} \propto A^{2} \propto \lambda_{\rho}^{-4}$. Similarly, for oblate sequences, the largest terms should be proportional to $\left(\eta_{, z z}\right)^{2} \propto A^{2} \lambda_{z}^{-4} \propto \lambda_{z}^{-3}$.

In Fig. 5 we show three representative configurations from a prolate sequence with $\lambda_{z}=0.8$, and we plot contours of the Riemann invariant $I$. Although there is a degree of self-similarity between the three solutions (note 

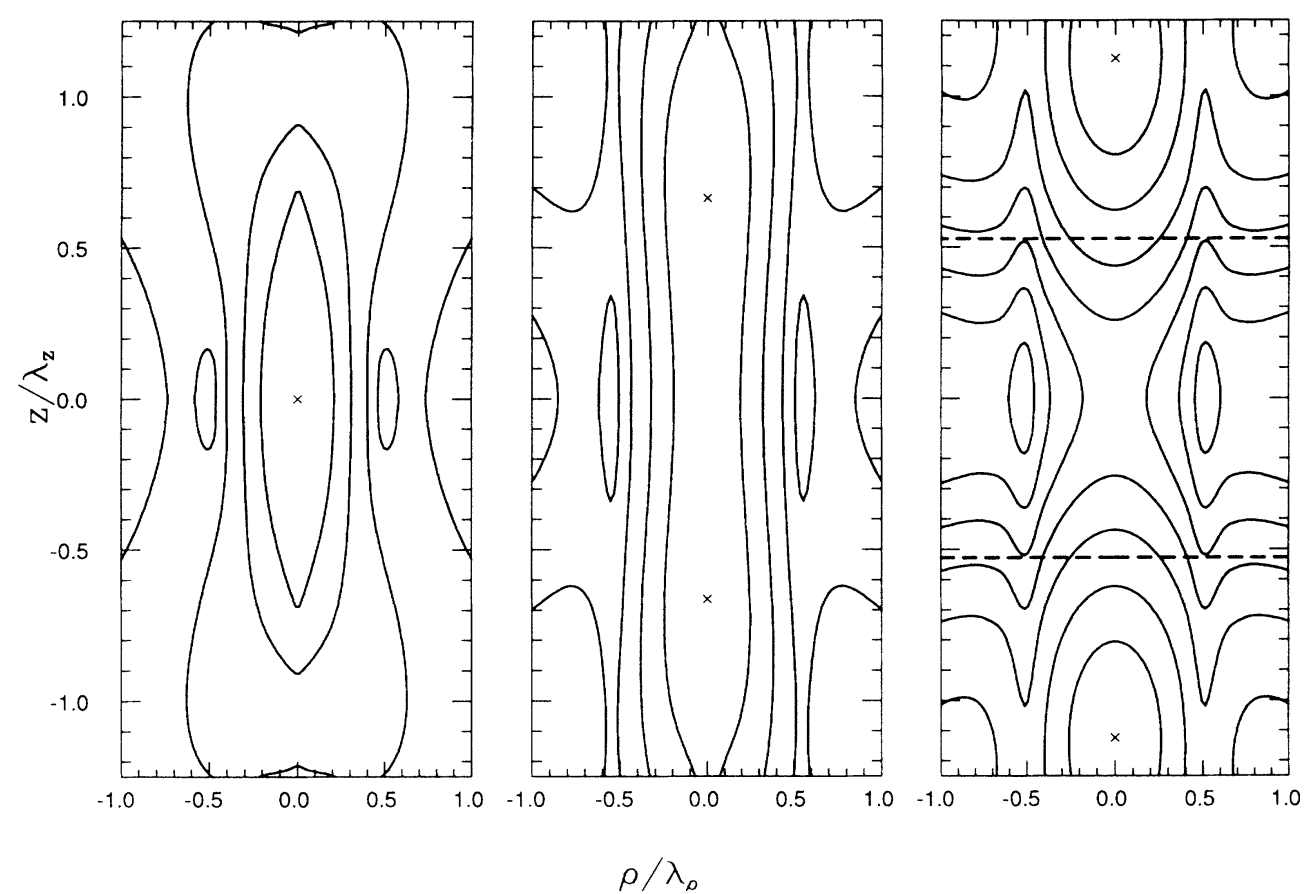

FIG. 5. Contours of the Riemann invariant $I$ are shown for a sequence of three wave packets each with characteristic length $\lambda_{z}=0.8$ and with characteristic widths $\lambda_{\rho}=0.8$ (left), $\lambda_{\rho}=0.064$ (center), and $\lambda_{\rho}=0.001$ (right). The highest contour level in each case is $I_{\max } / 2$ with each successive contour lower by a factor of 10 . The positions of the maxima are marked with an $\times$. In the far right frame, part of the apparent horizon is shown as a dashed line. See Table II.
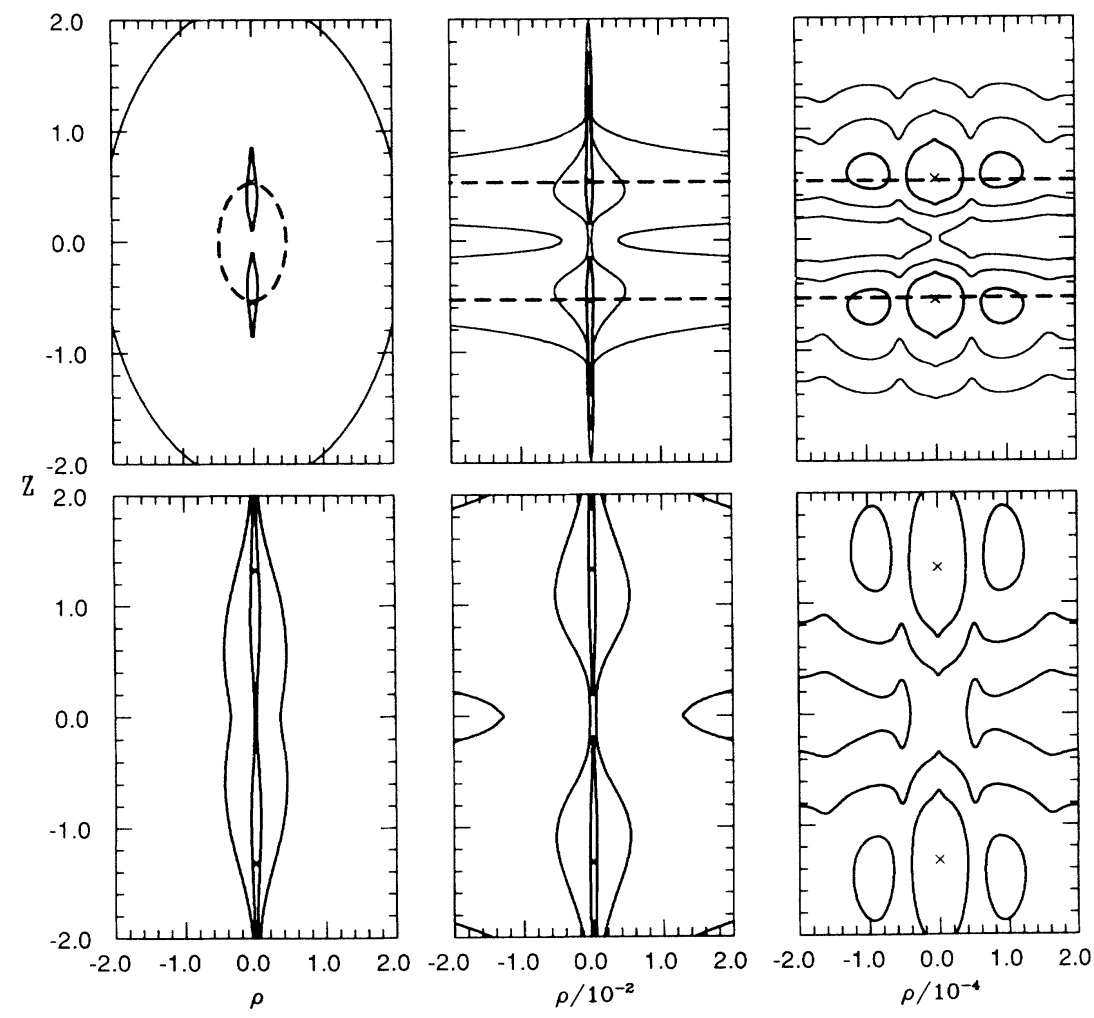

FIG. 6. Upper three frames detail a single, highly prolate Brill wave with $\lambda_{z}=0.45$ and $\lambda_{\rho}=1.0 \times 10^{-4}$, while the lower three show a solution with $\lambda_{z}=1.2$ and $\lambda_{\rho}=1.0 \times 10^{-4}$. The left frame in both rows spans a large range in both $\rho$ and $z$. The successive frames each zoom in by a factor of 100 on the region about the axis while keeping the $z$ range fixed. The contours of $I$, the Riemann invariant, are $I_{\max } / 10^{14}$ and $I_{\max } / 10^{16}$ in the left frames, $I_{\max } / 10^{6}, I_{\max } / 10^{10}$, and $I_{\max } / 10^{14}$ in the middle frames, and $I_{\max } / 10, I_{\max } / 10^{3}$, and $I_{\max } / 10^{6}$ in the right frames. The positions of the maxima of $I$ are noted by $\times$ 's. In each upper frame, the apparent horizon is indicated by the dashed line. See Table II. 

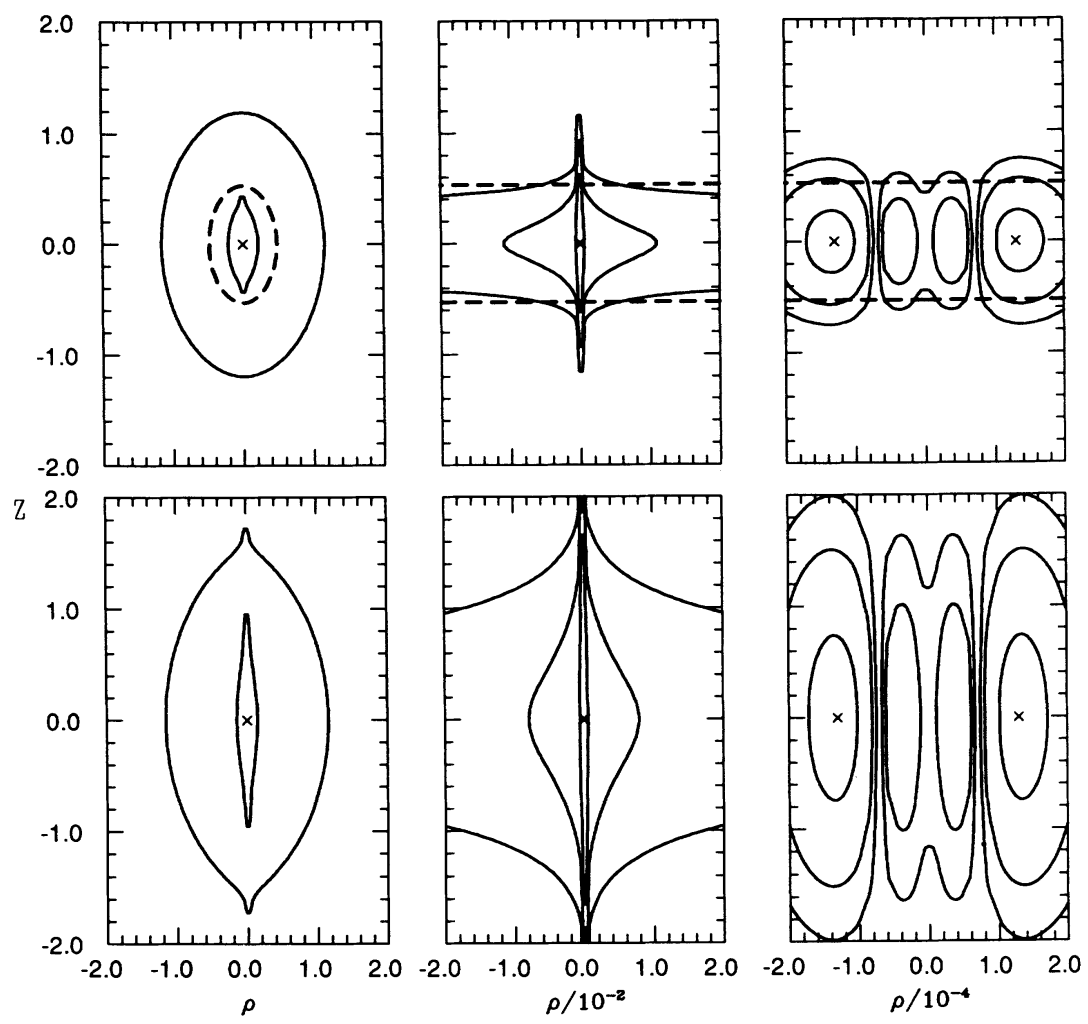

FIG. 7. Contours of the effective Brill mass density $D$ are shown for the same configurations depicted in Fig. 6 . The contours of $D$ are $D_{\max } / 2 \times 10^{6}$ and $D_{\max } / 2 \times 10^{8}$ in the left frames, $D_{\max } / 2 \times 10^{2}, D_{\max } / 2 \times 10^{4}$, and $D_{\max } / 2 \times 10^{6}$ in the middle frames, and $D_{\max } / 2.00, D_{\max } / 20.0$, and $D_{\max } / 200$. in the right frames. For $\lambda_{z}=0.45$ the maximum value of the Brill mass density is $D_{\max }=1.37 \times 10^{7}$ and, for $\lambda_{z}=1.2, D_{\max }=1.10 \times 10^{7}$. The positions of the maxima are noted by $\times$ 's. In each upper frame, the apparent horizon is indicated by the dashed line.

that our $\rho$ scale in each plot is in units of $\lambda_{\rho}$ ), the separation of the Riemann invariant maxima and the associated concentration of the contours are clearly seen. The third frame shows a configuration sufficiently compact that an apparent horizon forms. Although this horizon contains most of the mass energy of the slice-i.e., the proper area $\mathcal{A}$ of the apparent horizon satisfies $\mathcal{A} / 16 \pi M^{2} \sim 0.96-$ the two maxima of $I$ lie outside the apparent horizon. It is conceivable that in a real evolution the horizon would eventually envelop the maxima as they became singular; however, our prolate sequences do not show this behavior.

In Fig. 6 we demonstrate the multiscale structure in our solutions by "zooming in" in the $\rho$ direction on two solutions, one with a horizon and one without. We show three frames of $I$ contours for each. The structure of $I$ is quite similar in both cases, the main difference being the compactness of the structure in the $z$ direction. On the largest scale, the $I$ contours look roughly spherical for the $\lambda_{z}=0.45$ case, while they are still quite prolate for $\lambda_{z}=1.2$. Figure 7 shows contours of the Brill mass density [the integrand of Eq. (3.9)] for the initial-data sets illustrated in Fig. 6. For each of these highly prolate configurations, the maxima of the Brill mass density occurs along the equator very close to the axis. For the wave with $\lambda_{z}=0.45$, it is clear that a very high fraction of the mass is concentrated inside a quasi-isotropic coordinate radius of $0.5 \mathrm{M}$, while this is not obviously the case

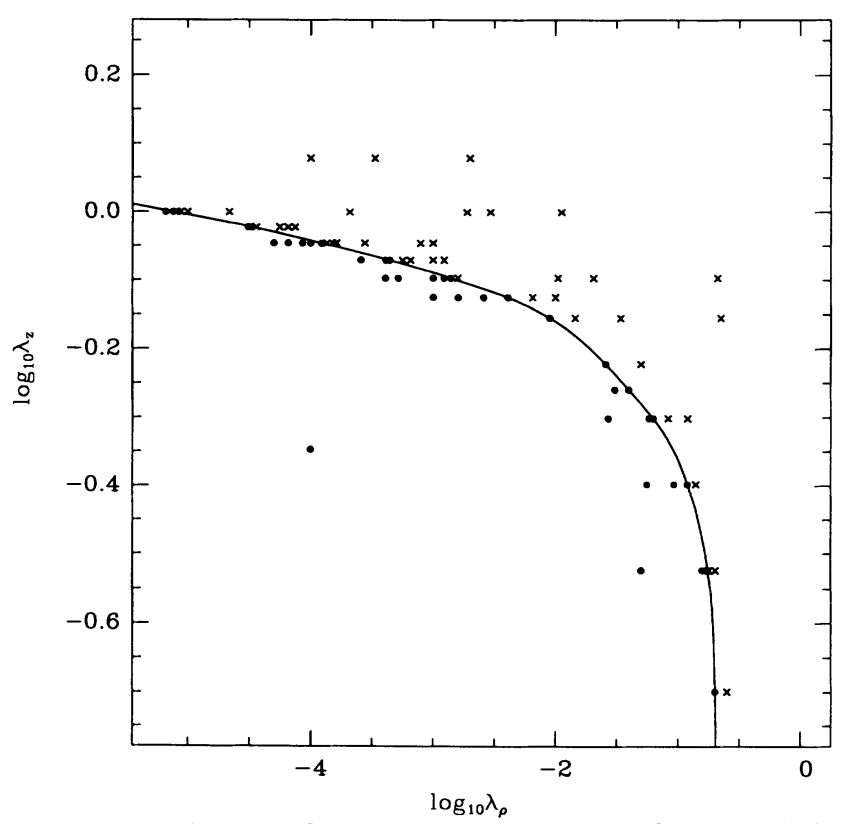

FIG. 8. Existence of an apparent horizon as a function of the compactness of a Brill wave is shown. For each configuration, parametrized by $\left(\lambda_{z}, \lambda_{\rho}\right)$, we indicate with a solid circle if an apparent horizon is found and with an $X$ if none is found. The line provides a rough spline fit to the boundary of the region where we find horizons. Note that the mass of each configuration is 1.0 in our units. 
TABLE II. Data for several Brill waves described by Eqs. (3.1)-(3.3) are given. Each wave packet is parametrized by its characteristic coordinate length $\lambda_{z}$, its characteristic coordinate width $\lambda_{\rho}$, and its amplitude chosen so that the wave's total mass is unity. We also list the maximum value $I_{\max }$ of the Riemann invariant, the position $z_{\max }$ along the axis at which this maximum value is achieved, the circumference of the minimum polar hoop surrounding $z_{\max }$, and whether or not an apparent horizon was found.

\begin{tabular}{lllllll}
\hline \hline$\lambda_{z} / M$ & $\lambda_{\rho} / M$ & Amplitude & $I_{\max } / M^{-4}$ & $z_{\max } / M$ & $\varrho_{\mathrm{pol}} / 4 \pi M$ & Horizon \\
\hline $4.5 \times 10^{-1}$ & $1.0 \times 10^{-4}$ & $2.87 \times 10^{8}$ & $4.03 \times 10^{14}$ & 0.546 & 1.022 & yes \\
$5.0 \times 10^{-1}$ & $5.8 \times 10^{-2}$ & $1.87 \times 10^{3}$ & $1.88 \times 10^{5}$ & 0.305 & 1.045 & yes \\
$8.0 \times 10^{-1}$ & $8.0 \times 10^{-1}$ & $1.05 \times 10^{1}$ & $6.06 \times 10^{3}$ & 0.00 & 0.000 & no \\
$8.0 \times 10^{-1}$ & $6.4 \times 10^{-2}$ & $1.29 \times 10^{3}$ & $3.11 \times 10^{5}$ & 0.532 & 1.024 & no \\
$8.0 \times 10^{-1}$ & $1.0 \times 10^{-3}$ & $3.19 \times 10^{6}$ & $4.00 \times 10^{11}$ & 0.899 & 1.111 & yes \\
$1.2 \times 10^{0}$ & $1.0 \times 10^{-4}$ & $2.55 \times 10^{8}$ & $6.74 \times 10^{16}$ & 1.323 & 1.237 & no \\
$2.0 \times 10^{0}$ & $2.0 \times 10^{-3}$ & $6.97 \times 10^{5}$ & $1.82 \times 10^{11}$ & 2.05 & 1.477 & no \\
\hline \hline
\end{tabular}

for the configuration with $\lambda_{z}=1.2$.

In Fig. 8 we delineate the existence or nonexistence of an apparent horizon in the initial-data set as a function of $\lambda_{z}$ and $\lambda_{\rho}$. As expected, if both wavelengths are small $(\lesssim 0.2)$, horizons always exist. Moreover, if there is a horizon in a data set with parameters $\lambda_{z 0}, \lambda_{\rho 0}$, we never find cases with $\lambda_{z} \leq \lambda_{z 0}$ and $\lambda_{\rho} \leq \lambda_{\rho 0}$ that do not have horizons. Similarly, if there is no horizon in a data set with parameters $\lambda_{z 0}, \lambda_{\rho 0}$, we never find horizons for cases with $\lambda_{z} \geq \lambda_{z 0}$ and $\lambda_{\rho} \geq \lambda_{\rho 0}$. In typical prolate sequences, when the horizon first appears it surrounds a large fraction of the mass $\mathcal{A} / 16 \pi M^{2} \gtrsim 0.90$, and this fraction increases toward unity as $\lambda_{\rho} \rightarrow 0$. We never find disjoint horizons or single horizons for sequences with $\lambda_{z}>1$.0. Our results indicate that one can choose an arbitrarily small value of $\lambda_{\rho}$ and always find a sufficiently large value of $\lambda_{z}$ such that the configuration has no apparent horizon.

Since in the pure-wave case the "source" of the gravitational field, the function $\eta$, does not have compact support, this example cannot test directly the hoop conjecture in its usual formulation. However, in order to characterize the prolate nature of our solutions, we can make a somewhat arbitrary polar-hoop definition (there seems to be no consistent way to define equatorial hoops) and draw a loose connection. First, we locate the point on the $z$ axis at which the invariant $I$ obtains its maximum value. Then we search for the hoop with the smallest proper length that hits the axis above the maximum. In Table II we give sample parameters and minimum polar-hoop circumferences for some of the highly prolate configurations discussed above. We find that if the normalized hoop length is considerably larger than the hoop conjecture cutoff, say $C_{\text {pol }} / 4 \pi M \gtrsim 1.2$, we never find horizons. However, particularly at low field strengths when the peak of the invariant is near the origin, the polarhoop circumference can be much smaller than 1.0 without a horizon. Since the hoop is enclosing only a very small fraction of the wave mass in this circumstance, it is difficult to make quantitative contact with the hoop conjecture for these cases.

To summarize, we have demonstrated that (a) sequences of Brill waves with prolate profiles can be constructed in which the gravitational tidal field diverges, (b) the location of the point of strongest tidal field can be well away from the bulk of the wave's mass energy, (c) one can choose an arbitrarily high value for the tidal field and always find a configuration with that field strength but without an apparent horizon, and (d) the presence or absence of apparent horizons is qualitatively consistent with the hoop conjecture. If the configuration is sufficiently long, apparent horizons are never found. The extreme members of our wave sequences may be good candidates for forming naked singularities. They should be employed as initial data in an evolution code to see if they indeed form singularities without apparent horizons. Based on experience with collisionless matter, we anticipate that time-dependent simulations (at least those using maximal slices) should behave in this fashion.

\section{ACKNOWLEDGMENTS}

We acknowledge useful conversations with G. B. Cook, L. S. Finn, J. L. Friedman, and K. S. Thorne. This research was supported in part by NSF Grants Nos. AST 90-15451 and PHY 90-07834 and NASA Grant No. NAGW-2364 at Cornell University. A. A. was supported by the NSF Division of Advanced Scientific Computing. Computations in support of this work were performed at the Cornell National Supercomputing Facility, which is supported in part by the National Science Foundation, IBM Corporation, New York State, and the Cornell Research Institute.
[1] S. L. Shapiro and S. A. Teukolsky, Phys. Rev. Lett. 66, 994 (1991); Am. Sci. 79 (4), 330 (1991).

[2] S. L. Shapiro and S. A. Teukolsky, Phys. Rev. D 45, 2006 (1992).

[3] D. Goldwirth, T. Piran, and A. Ori, in Frontiers in Numer- ical Relativity, edited by C. R. Evans, L. S. Finn, and D. W. Hobill (Cambridge University Press, Cambridge, England, 1989).

[4] T. A. Apostolatos and K. S. Thorne (unpublished).

[5] K. S. Thorne, in Magic Without Magic: John Archibald 
Wheeler, edited by J. Klauder (Freeman, San Francisco, 1972), p. 231.

[6] T. Nakamura, S. L. Shapiro, and S. A. Teukolsky, Phys. Rev. D 38, 2972 (1988).

[7] J. L. Friedman, Nature 351, 269 (1991).

[8] R. M. Wald and V. Iyer, Phys. Rev. D 44, R3719 (1991).

[9] S. W. Hawking and G. F. R. Ellis, The Large Scale Structure of Space-Time (Cambridge University Press, Cambridge, England, 1973), p. 319.

[10] N. T. Bishop, Gen. Relativ. Gravit. 14, 717 (1982).

[11] N. T. Bishop, Gen. Relativ. Gravit. 16, 589 (1984).

[12] A. Cadez, Ann. Phys. (N.Y.) 83, 449 (1974).

[13] A. P. Lightman, W. H. Press, R. H. Price, and S. A. Teukolsky, Problem Book in Relativity and Gravitation (Princeton University Press, Princeton, 1975), p. 588.

[14] J. Wojtkiewicz, Phys. Rev. D 41, 1867 (1990).

[15] A. Einstein and N. Rosen, J. Franklin Inst. 223, 43 (1937).

[16] D. R. Brill, Ann. Phys. (N.Y.) 7, 466 (1959).

[17] J. Weber and J. A. Wheeler, Rev. Mod. Phys. 29, 509
(1957).

[18] K. Eppley, in Sources of Gravitational Radiation, edited by L. Smarr (Cambridge University Press, Cambridge, England, 1979).

[19] C. R. Evans, in Dynamical Spacetimes and Numerical Relativity, edited by J. Centrella (Cambridge University Press, Cambridge, England, 1986), p. 3; A. M. Abrahams, in Frontiers in Numerical Relativity [3], p. 110.

[20] R. Beig and N. Ó Murchadha, Phys. Rev. Lett. 66, 2421 (1991).

[21] R. Arnowitt, S. Deser, and C. W. Misner, in Gravitation: An Introduction to Current Research, edited by L. Witten (Wiley, New York, 1962).

[22] MathTensor ${ }^{\circledR}$ is a Mathematica-based tensor analysis package developed by L. Parker and S. Christensen.

[23] S. L. Shapiro and S. A. Teukolsky, Phys. Rev. D 45, 2739 (1992).

[24] T. Piran, P. N. Safier, and R. F. Stark, Phys. Rev. D 32, 3101 (1985). 